\title{
Larva migrans cutánea: diagnóstico de sospecha y tratamiento en Atención Primaria
}

\author{
C. S. Varela Castro, Ma a Varela Cerdeira, M. L. Pascual Martín* \\ Residente de M edicina Familiar y Comunitaria. *Especialista M edicina Familiar y \\ Comunitaria. Centro de Salud Universitario de la UAM Chopera 1. Alcobendas. \\ Unidad Docente de Medicina Familiar y Comunitaria Área 5. Madrid
}

\section{RESUMEN}

Larva migrans cutánea es un término clínico que designa una erupción dérmica de carácter li neal y serpiginoso, producida por larvas de gusanos nematelmintos. Algunos autores lo hacen sinó nimo de erupción serpiginosa (creeping eruption).

El Ancylostoma Braziliense es origen de la más frecuente y clínicamente más característica larva migrans. Se observa, principalmente, en América Central y Sur, y en los estados sureños de Estados Unidos. Dado el apogeo y la alta incidencia de viajes de ocio en nuestro medio, la frecuencia de este tipo de parasitosis se ha incrementado en nuestras consultas y puesto que su diagnóstico es fundamentalmente clínico, es recomendable su co nocimiento en Atención Primaria.

Palabras clave: Parasitosis. Larva migrans cutánea. Tratamiento. Prurito.
Cutaneous larva migrans: diagnosis and treatment in Primary Health Care

\begin{abstract}
Cutaneous larva migrans is a clinical term that designates a dermal eruption of lineal and serpigi nous character, caused by larvas of nematelmintos worms. Some authors make it synonymous of serpi ginous eruption (creeping eruption). The Ancylostoma Braziliense is the origin of the most frequent and the most clinically characteristic larva mi grans. It is observed, mainly, in central and South America, and in the southern states of United Sta tes. Given the high incidence of leisure trips in our environment, the frequency of this parasitic disease has been increased in our consultations and since its diagnosis is fundamentally clinical, it is advisa ble its knowledge in Primary Health Care.
\end{abstract}

Key words: Parasitic skin diseases. Cutaneous larva migrans. Treatment. Pruritus.

\section{INTRODUCCIÓN}

La larva migrans cutánea (LMC) es una parasitosis, caracterizada por lesiones cutáneas serpiginosas, endémica en climas cálidos y húmedos de áreas tropicales y subtropicales, pero cada vez más evidente en otras áreas dada la frecuencia de turistas y viajes de placer a países exóticos.

El agente etiológico más frecuente es el Ancy lostoma Braziliense, un helminto que como gusano adulto, vive en el intestino de gatos, perros y felinos salvajes. Por sus heces se eliminan numerosos huevos que sobreviven en terreno húmedo y arenoso (playas, jardines), donde se convierten en larvas con capacidad infectante. Al contacto con la piel, la penetran y se alojan en ella. Inicialmente dan lugar a una lesión papular eritematosa, más frecuentemente localizada en los pies. Después de un tiempo variable, la larva emigra labrando un trayecto intraepidérmico, que se manifiesta como lesión eritema- 
tosa, pruriginosa, que sobreeleva la piel, y crece desde algunos milímetros a 2-5 centímetros al día, localizada sobre todo en los pies, nalgas y muslos. Algún caso clínico se ha manifestado sólo en forma de foliculitis ${ }^{1}$.

La erupción cutánea puede acompañarse de eosinofilia, elevación de la IgE e incluso infiltrados pulmonares eosinofílicos (síndrome de Loefller).

Es una enfermedad autolimitada, el ser humano es huésped terminal, donde la larva no puede llegar a adulto, termina muriendo y desapareciendo en 1 a 6 meses $^{2}$.

\section{CASO CLÍNICO}

Presentamos el caso de un varón de 21 años de edad, sin antecedentes clínicos de interés, que consultó a su médico de Atención Primaria por intenso prurito en la zona interdigital y cara plantar de ambos pies, de 4 días de evolución. No refería fiebre ni ninguna otra manifestación sistémica. Seis días antes había regresado a España tras un viaje a América Central de dos semanas de duración.

En la primera exploración física que realizamos presentaba en las zonas interdigitales y planta de ambos pies varias lesiones pápulo-eritematosas, con superficie excoriada y costrosa, y otras en forma de surcos serpiginosos intraepidérmicos de $30 \mathrm{~mm}$ de longitud (Figs. 1 y 2 ).

Pruebas complementarias: hemograma, función hepática y radiografía de tórax, sin alteraciones.

Se procedió a tratamiento con albendazol $200 \mathrm{mg}$ cada 12 horas durante cinco días, con evolución favorable, ya que desaparecieron progresivamente las lesiones en el transcurso de diez días.

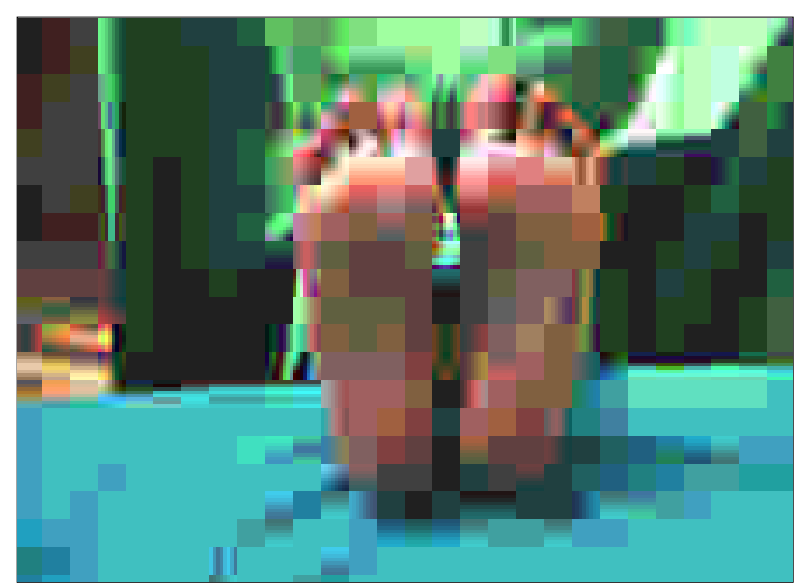

Figura 1

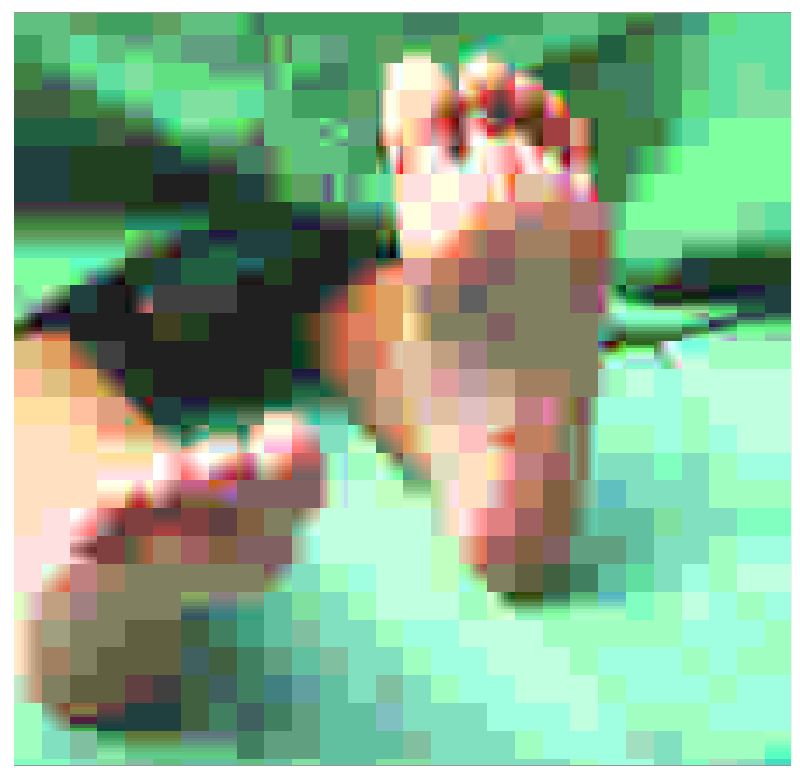

Figura 2

\section{DISCUSIÓN}

Las enfermedades exóticas importadas de tipo infeccioso y parasitario están adquiriendo una prevalencia creciente en los países europeos debido a los movimientos migratorios y al aumento del turismo. La mayor parte de estas enfermedades cursan con síntomas cutáneos y entre ellas se encuentra la larva migrans cutánea. Por ello, recomendamos conocer las formas de diagnóstico y tratamiento de este helminto.

Su diagnóstico es fundamentalmente clínico, basado en la evidencia de las lesiones cutáneas con típicos trayectos serpiginosos y muy pruriginosos. Localizados fundamentalmente en zonas donde el grosor de la piel es más delgado como los espacios interdigitales de los pies, nalgas y muslos. Una anamnesis orientada a preguntar al paciente sobre viajes recientes a países tropicales nos ayudará en el diagnóstico.

La biopsia de la lesión es extremadamente difícil pues con frecuencia, el parásito se localiza más allá de las lesiones visibles. Las pruebas hematológicas pueden, en algunas ocasiones, revelar eosinofilia o discreto incremento de la $\operatorname{IgE}^{2,3}$.

Algunos autores proponen la epiluminiscencia microscópica como diagnóstico de confirmación y otros ELISA para detectar IgG específica ${ }^{4}$.

Esta dermatosis plantea diagnóstico diferencial con otras parasitosis: larva migrans viscerale, erupciones por Strongyloides Stercolaris, nódulos subcutáneos o granulomas debidos a otras especies y cuadros clínicos de miasis y con otras enfermedades más comunes como dermatitis alérgicas de contacto, sarna, pioderma o urticaria facticia ${ }^{2}$.

Aunque normalmente la larva desaparece por sí sola en uno a seis meses, el intenso prurito y la sen- 
sación desagradable que tiene el paciente de la larva arrastrándose a través de la piel, nos obligan a prescribir un tratamiento adecuado.

Existen varias opciones terapéuticas tópicas y sistémicas. Clásicamente se han venido utilizando la crioterapia en el extremo de la lesión que progresa, pero con el inconveniente de la dificultad de identificar la posición exacta del parásito ${ }^{5}$. El tiabendazol tópico al $10 \%$ puede utilizarse en algunas lesiones no muy extendidas, debe administrarse repetidamente a lo largo del día y exige especial seguimiento para evitar recurrencias. Algunos autores sugieren el uso tópico de crema con $2 \%$ de gammexano; ungüento con $25 \%$ de piperazina o metrifonato. El uso sistémico de tiabendazol $50 \mathrm{mg}$ al día, durante dos a cinco días, podría ser utilizado en el caso de lesiones extensas, pero está contraindicado por una baja tolerabilidad de sus efectos secundarios. Flubendazol $200 \mathrm{mg}$ al día, durante cinco días, está actualmente en fase experimental, y podría presentarse como una posibilidad de futuro ${ }^{6}$.
Actualmente, una alternativa válida al tratamiento convencional es el uso de albendazol 400$800 \mathrm{mg} /$ día, durante un periodo que puede variar entre dos y siete días, y según los últimos estudios parece alzarse como la droga de primera elección ${ }^{6}$.

Como medidas preventivas en Atención Primaria debemos recomendar a nuestros pacientes un calzado adecuado para evitar el contacto con la larva en playas tropicales.

Por ser una enfermedad poco habitual en nuestro medio, no debemos perder la capacidad de sospecha y debe estar presente en los diagnósticos diferenciales en Atención Primaria.

\section{CORRESPONDENCIA:}

C. Sylvia Varela Castro

C/ Santiago de Compostela $n^{\circ} 84,9^{\circ} \mathrm{G}$

28035 Madrid

\section{Bibliografía}

1. Caumes E. Treatment of cutaneous larva migrans. Clin Infect Dis 2000; 30: 811-4.

2. Dandén Tello E, Oñate Cuchet MJ. Dermatosis causadas por artrópodos, helmintos y protozoos. En: Iglesias Díez L. Tratado de Dermatología. $1^{a}$ ed. Madrid: Luzán, 1994. p. 55-90.

3. García Pérez A. Dermatosis por parásitos metazoos. En: García Pérez A. Dermatología clínica. $4^{\mathrm{a}}$ ed. Salamanca: Gráficas Cervantes, 1987. p. 31-8.

4. Veraldi S, Schianchi R, Carrera C. Epiluminiscence micros- copy in cutaneous larva migrans. Acta Derm Venereol 2000; 80 (3): 233.

5. Bouchaud O, Houzé S, Schiemann R, Durand R, Ralaimazava $\mathrm{P}$, Ruggeri $\mathrm{C}$, et al. Cutaneous larva migrans in travelers; a prospective study, with Assessment of therapy with Ivermectin. Clin Infect Dis 2000; 31: 493-8.

6. Albanese G, Venturi C, Galbiati G. Treatment of cutaneous larva migrans (creeping eruption): a comparison between albendazole and traditional therapy. Intern J Dermatol 2001; 40: $67-71$. 